\title{
Medición del diámetro de la vaina del nervio óptico por ultrasonografía versus tomografía simple de cráneo en pacientes con trauma craneoencefálico
}

Measurement of the diameter of the optical nerve sheath by ultrasonography versus

head computed tomography in patients with traumatic brain injury

Medição do diâmetro da bainha do nervo óptico medido por ultrassonografia versus

tomografia craneal em pacientes com trauma craneoenefálico

Roberto Emmanuel Islas Ávila, ${ }^{*}$ Valeria Leticia Coria Ladrón de Guevara, ${ }^{*}$ Felipe de Jesús Montelongo, ${ }^{*}$ María Magdalena Reyes Pérez, ${ }^{*}$ Aurea Carmona Domínguez, ${ }^{*}$ Araceli Suárez Suárez*

\section{RESUMEN}

Introducción: El trauma craneoencefálico es una causa importante de muerte y secuelas en nuestro país. La causa principal de muerte para estos pacientes es el aumento de la presión intracraneal. Existen diferentes métodos de monitorización de la presión intracraneal, puede ser por métodos invasivos y no invasivos. Entre los métodos no invasivos destaca por su accesibilidad la medición de la vaina del nervio óptico por ultrasonografía a la cabecera del paciente. Nuestro estudio pretende encontrar la distancia adecuada en milímetros a partir del globo ocular con la cual la medición del diámetro de la vaina del nervio óptico por ultrasonografía es más precisa si se compara con la medición en un estudio de tomografía simple de cráneo.

Material y métodos: Se realizó un estudio de tipo observacional, prospectivo, transversal y analítico entre pacientes que ingresaran al Servicio de Terapia Intensiva Neurológica del Hospital General de Ecatepec Las Américas y que cumplieran con los criterios de ingreso en el periodo comprendido entre el $1^{\circ}$ de noviembre de 2018 y el 31 de enero de 2019. Se compararon las medidas del diámetro de la vaina del nervio óptico a 3, 6 y $9 \mathrm{~mm}$ de cada globo ocular por ultrasonografía contra las medidas a las mismas distancias por tomografía simple de cráneo de cada uno de los pacientes.

Resultados: No se encontró diferencia estadísticamente significativa al comparar las mediciones por ultrasonografía contra tomografía simple de cráneo en ninguna de las tres distancias establecidas $(3,6$ y $9 \mathrm{~mm}$ ) con una $p>0.05$ en todos los casos. La distancia a la cual se encontró una menor diferencia entre las medidas fue a $3 \mathrm{~mm}$ a partir del globo ocular, esto sin significancia estadística. El trauma craneoencefálico en la población estudiada afecta principalmente a hombres jóvenes en edad productiva. La lesión que se encontró con mayor frecuencia en los estudios de tomografía simple de cráneo de los pacientes estudiados fue la hemorragia subaracnoidea.

Conclusiones: La medición de la vaina del nervio óptico a 3, 6 y $9 \mathrm{~mm}$ a partir del globo ocular por ultrasonografía no muestra diferencia estadísticamente significativa si la comparamos con la medición de la vaina del nervio óptico mediante tomografía simple de cráneo en pacientes con trauma craneoencefálico severo. Sin embargo, la distancia que demuestra una menor diferencia entre ambas medidas es a $3 \mathrm{~mm}$, la cual es la distancia ya establecida para realizar la medición en la bibliografía actual.

Palabras clave: Medición, nervio óptico, vaina, ultrasonografía, tomografía simple de cráneo, traumatismo craneoencefálico.

\section{ABSTRACT}

Introduction: Traumatic brain injury is a cause of death and neurological sequelae in Mexico. The main cause of death for these group of patients is intracranial hypertension. There are invasive and non-invasive intracranial pressure monitoring techniques. Within the non-invasive techniques group, perhaps the most accessible one could be the bed-side measurement of the diameter of the optic nerve sheath. Our study pretends to find the most precise distance in millimeters to measure de optic nerve sheath compared to measurements performed by cranial computed tomography.

Material and methods: A prospective, observational, transversal, analytical study was made with patients admitted in the Neurologic Intensive Care Unit from the Hospital General de Ecatepec Las Americas, whose met the inclusion criteria. The study was performed between November the $1^{\text {st }}$ of 2018 and January the $31^{\text {th }}$ of 2019. We compared the diameter of the optic nerve sheath

* Hospital General Ecatepec Las Américas del Instituto de Salud del Estado de México. Estado de México, México.

Recepción: 30/08/2019. Aceptación: 30/07/2020.

www.medigraphic.com/medicinacritica measured by ultrasonography at 3, 6 and $9 \mathrm{~mm}$ distance from each eye-ball versus the sizes obtained by cranial tomography at the same distances.

Results: No statistically significant difference was found by comparing ultrasonography acquired versus cranial tomography acquired optical nerve diameters, at none of the three mentioned distances $(3,6$ and $9 \mathrm{~mm})$ with a $p$ value $>0.05$ for all of them. The minor difference was found at $3 \mathrm{~mm}$ from the eye ball, but again, without statistical significance. Most of the studied patients were economically active men, and the most frequent injury found by cranial tomography was subarachnoid hemorrhage.

Conclusions: There is no statistically significant difference between the tomography measurement of optical nerve at 3, 6 and $9 \mathrm{~mm}$ and the measure of the optic nerve sheath at the same distances, in severe traumatic brain injury patients. Nevertheless, at $3 \mathrm{~mm}$ distance from the eyeball the minor difference was found between both techniques, the same distance traditionally described in consulted bibliography.

Keywords: Measurement, optic nerve, sheath, ultrasonography, cranial tomography, traumatic brain injury.

\section{RESUMO}

Introdução: O trauma cranioencefálico é uma importante causa de morte e sequelas em nosso meio. A principal causa de morte desses pacientes é o aumento da pressão intracraniana. Existem diferentes métodos de monitoramento da pressão intracraniana, podendo ser por métodos invasivos e não invasivos. Dentre os métodos não invasivos, a medida da bainha do nervo óptico por ultrassonografia à beira do leito se destaca pela acessibilidade. Nosso estudo visa encontrar a distância adequada em milímetros do globo ocular com a qual a medida do diâmetro da bainha do nervo óptico pela ultrassonografia seja mais precisa quando comparada à medida em um estudo de tomografia craneal.

Material e métodos: Foi realizado um estudo observacional, prospectivo, transversal e analítico em pacientes internados no serviço de terapia intensiva neurológica do Hospital Geral de Ecatepec Las Américas e que atendiam aos critérios de admissão. No período de 10 de novembro de 2018 a 31 de janeiro de 2019. As medidas do diâmetro da bainha do nervo óptico em 3, 6 e 9 mm de cada globo ocular medidas por ultrassonografia foram comparadas com as medidas de as mesmas distâncias por tomografia craneal de cada um dos pacientes.

Resultados: Não foi encontrada diferença estatisticamente significante ao comparar as medidas da ultrassonografia com a tomografia craneal em nenhuma das 3 distâncias estabelecidas $(3,6$ e $9 \mathrm{~mm}$ ) com $p>0.05$ em todos os casos. A distância em que foi encontrada a menor diferença entre as medidas foi de 3 $\mathrm{mm}$ do globo ocular, sem significância estatística. O trauma cranioencefálico na população estudada afeta principalmente homens jovens em idade produtiva. A hemorragia subaracnóidea foi a lesão mais freqüentemente encontrada nos estudos de tomografia craneal dos pacientes estudados.

Conclusões: A medida da bainha do nervo óptico a 3, 6 e 9 mm do globo ocular pela ultrassonografia não apresenta diferença estatisticamente significativa quando comparada com a medida da bainha do nervo óptico pela tomografia craneal em pacientes com trauma cranioencefálico grave. Porém, a distância que mostra a menor diferença entre as duas medidas é de $3 \mathrm{~mm}$, que é a distância já estabelecida para realizar a medida na bibliografia atual.

Palavras-chave: Medição, nervo óptico, bainha, ultrassonografia, tomografia craneal, traumatismo craneoencefálico.

\section{INTRODUCCIÓN}

El aumento de la presión intracraneal (PIC) es una causa importante de daño cerebral y su asociación con un 
pronóstico desfavorable ha sido ampliamente demostrada. El neuromonitoreo y el tratamiento oportuno del aumento de la presión intracraneal son la piedra angular en el manejo de pacientes neurocríticos. Desde hace muchos años la medición de la PIC por medios invasivos se considera el estándar de oro. Sin embargo, el monitoreo de la presión intracraneal por métodos invasivos se encuentra lejos de ser seguro, las complicaciones como las hemorragias y las infecciones alcanzan de 1 al $7 \%$ de los casos, llevando a la búsqueda y desarrollo de alternativas no invasivas. ${ }^{1}$ El riesgo de infecciones se incrementa con el tiempo de permanencia de los dispositivos. Además, la colocación de los dispositivos de monitorización invasiva debe realizarse por neurocirujanos o equipo médico de terapia intensiva especialmente entrenado, lo cual es una limitante para su uso. ${ }^{2}$ Varios métodos no invasivos de monitorización de la PIC se encuentran en proceso de investigación, aún no disponibles para su aplicación clínica.

\section{TRAUMA CRANEOENCEFÁLICO}

El trauma craneoencefálico (TCE) es cualquier lesión estructural o funcional del cráneo y/o su contenido secundario a un intercambio brusco de energía mecánica, y al menos alguno de los siguientes elementos: alteración de la conciencia y/o amnesia debida al trauma, cambios neurológicos o neurofisiológicos, o diagnóstico de fractura de cráneo o lesiones intracraneales atribuibles al trauma o la ocurrencia de muerte resultante del trauma que incluya los diagnósticos de lesión de la cabeza y/o lesión cerebral traumática entre las causas que produjeron la muerte. ${ }^{3,4}$

El trauma craneoencefálico es el tipo más común de trauma que se atiende en los servicios de emergencia de los Estados Unidos, con casi un millón de casos tratados anualmente. Muchos pacientes con trauma craneoencefálico grave mueren antes de llegar al hospital, y por lo menos $90 \%$ de las muertes prehospitalarias relacionadas al trauma involucran al trauma craneoencefálico. ${ }^{5}$ En Iberoamérica la incidencia de trauma craneoencefálico es de 200 a 400 por cada 100,000 habitantes por año, y es más frecuente en el sexo masculino, con una relación 2:1 a 3:1, afectando a la población joven económicamente activa. La mortalidad ronda $30 \%$ en los centros especializados en trauma. ${ }^{6}$ En México, el TCE ocupa la cuarta causa de muerte, los accidentes de tráfico contribuyen con $75 \%$ y el resto es debido a muertes violentas, caídas y lesiones deportivas. ${ }^{7}$

EI TCE grave representa $10 \%$ de todos los traumatismos craneoencefálicos, pero es el que contribuye en mayor proporción a los casos de fallecimientos, secuelas y el que incrementa los costos de la atención. Entre estos pacientes la causa principal de fallecimiento es la hipertensión intracraneal $(\mathrm{HIC}){ }^{8}$
Fisiopatológicamente el trauma craneoencefálico cursa con dos fases. La primera debida al impacto directo: fractura, contusión, hemorragia puntiforme y hemorragia subaracnoidea; y la segunda debida a edema, hipoxia y hemorragia subsecuente. El trauma directo produce contusión del parénquima cerebral y lesión axonal difusa en la sustancia blanca cerebral y del tronco encefálico. La lesión primaria desencadena una serie de alteraciones en el metabolismo cerebral que comprometen la hemodinamia intracraneal y la homeostasis iónica. El daño al parénquima cerebral se acompaña de isquemia e hipoxia. ${ }^{4}$

Existen diferentes formas de clasificar al trauma craneoencefálico. Puede ser por mecanismo del trauma, por severidad o por la morfología de las lesiones.

Con base en el mecanismo del trauma, el TCE se clasifica en abierto y cerrado. El trauma abierto se define por la penetración de la duramadre, causada comúnmente por proyectiles y esquirlas. El trauma abierto se asocia a mayor mortalidad comparado con el TCE cerrado (88 vs. $32 \%) .{ }^{9}$ El trauma cerrado es aquél en el que no existe penetración de la duramadre, y su causa más frecuente son los accidentes de vehículo automotor. Otras menos frecuentes son caídas y trauma directo con objeto contundente. ${ }^{4}$

Para clasificar el trauma craneoencefálico por severidad de la lesión se usa la escala de coma de Glasgow (GCS) como una medida clínica objetiva de lesión cerebral. Una GCS con puntaje de ocho o menos se ha convertido en la definición generalmente aceptada de coma o lesión cerebral grave. Los pacientes con trauma cerebral y con una GCS de 9 a 12 se clasifican como «moderados» y aquéllos con un puntaje de 13 a 15 se clasifican como «leves». ${ }^{5}$ Se estima que aproximadamente $80 \%$ de los traumatismos craneoencefálicos son de intensidad leve y el restante $20 \%$ se divide entre traumatismos moderados y graves. 7,8

La clasificación por morfología se relaciona con las lesiones que se pueden incluir, ya sean fracturas de cráneo y/o lesiones intracraneales como contusiones, hematomas, hemorragias, lesiones difusas y el edema resultante. ${ }^{5}$

\section{PRESIÓN INTRACRANEAL}

La presión intracraneal (PIC) es la presión requerida para desplazar un volumen determinado en la caja craneal. Su aumento representa un cambio de presión en respuesta a los cambios del volumen. El aumento de volumen intracraneal puede producirse por:

- Presencia de un hematoma intraaxial o extraaxial.

- Aumento del volumen del líquido cerebro espinal (LCE).

- Edema citotóxico, edema neurotóxico o edema vasogénico. 
- Aumento del volumen sanguíneo cerebral (VSC).

- Patología vascular arterial o venosa. ${ }^{10}$

La presión de perfusión cerebral (PPC) está determinada por la diferencia entre la presión arterial media (PAM) y la presión intracraneal $(\mathrm{PIC}):$ PPC = PAM-PIC. Cuando está conservado el mecanismo de autorregulación, la PPC puede oscilar entre 50 y $150 \mathrm{mmHg} \sin$ generar variaciones significativas en el flujo sanguíneo cerebral (FSC). El cráneo es un continente rígido, no distensible y la presión en su interior está regida por las variaciones del volumen de su contenido. El volumen de la cavidad intracraneal es de 1,200 a 1,400 mL. En condiciones normales la PIC es inferior a $10 \mathrm{mmHg}$ y está determinada por el volumen cerebral $(85 \%=$ 1,020-1,190 mL), líquido cefalorraquídeo (LCR) (10\% $=120-140 \mathrm{~mL})$ y volumen sanguíneo $(5 \%=60-70 \mathrm{~mL}$, de éste $70 \%$ venoso, $15 \%$ linfático y $15 \%$ arterial). ${ }^{9-11}$

La ley de Monro-Kellie expresa que la PIC depende de las variaciones del volumen de los elementos intracraneales, y que dentro de este espacio cerrado no distensible la variación de uno de los volúmenes genera cambios en sentido opuesto en los restantes. El aumento del volumen cerebral producirá reducción del volumen sanguíneo venoso y del LCR con el fin de mantener la PIC.4,5,11,12 Inicialmente se reduce el volumen venoso, pero su capacidad de adaptarse se agota mucho antes que la del LCR ante el aumento progresivo y sostenido de la PIC, mientras que el volumen arterial es el menos complaciente, e incluso puede generar aumento de la PIC. En estas condiciones, la reducción de la PPC induce vasodilatación arterial que incrementa el FSC y por tanto, del volumen sanguíneo cerebral (VSC) provocando la elevación de la PIC. ${ }^{4}$

El aumento de la PIC somete al tallo encefálico a hipoperfusión e isquemia, lo cual desencadena un aumento paralelo de la actividad del sistema nervioso autónomo simpático y parasimpático, que trata de aumentar el volumen latido y la presión arterial a niveles que superen la presión ejercida sobre el tallo encefálico en aras de vencer la resistencia vascular al FSC generada por el aumento de la PIC. Esta respuesta fisiológica ante la elevación de la PIC conocida como fenómeno o ley de Cushing se caracteriza clínicamente por hipertensión arterial sistémica y bradicardia. ${ }^{4}$

El aumento de la presión intracraneal (PIC) puede reducir la perfusión cerebral y causar o exacerbar la isquemia. Las presiones por arriba de $20 \mathrm{mmHg}$, particularmente si son prolongadas y refractarias al tratamiento, están asociadas con un mal pronóstico. ${ }^{5}$

\section{HIPERTENSIÓN INTRACRANEAL}

Se refiere al aumento de la presión intracraneal por arriba de $15 \mathrm{mmHg}$ de forma sostenida o mayor de 20
$\mathrm{mmHg}$, dependiendo de la bibliografía que se consulte. En las guías actuales se considera una presión intracraneal por arriba de $22 \mathrm{mmHg}$ para el inicio del tratamiento. Existen diversas condiciones que pueden llevar a una elevación de la presión intracraneal dependiendo del padecimiento, ya sea agudo o crónico. ${ }^{13}$

La hipertensión intracraneal traumática es una complicación frecuente y dañina del trauma cerebral que contribuye de forma importante al daño cerebral secundario, su severidad y duración se han correlacionado con un pronóstico fatal. ${ }^{14-16}$ Las causas principales de aumento de la presión intracraneal se pueden resumir en traumatismo craneoencefálico, hemorragia subaracnoidea, trombosis venosa cerebral, encefalopatía anoxicoisquémica, tumores cerebrales, hematomas, hipertensión intracraneal idiopática, encefalopatía hepática aguda, síndrome hiperosmolar agudo, encefalopatía hipertensiva, síndrome de Reye y craneosinostosis.

La relación entre el volumen intracraneal y la presión intracraneal es exponencial. De forma inicial la presión aumenta discretamente con incrementos de volumen, pero cuando los mecanismos de autorregulación del sistema se exceden, la presión intracraneal aumenta abruptamente. Esto explica el rápido deterioro que con frecuencia se observa en pacientes con un hematoma intracraneal postraumático. ${ }^{13}$

Los eventos intracraneales y sistémicos contribuyen de forma conjunta a elevar la presión intracraneal después de un trauma craneoencefálico. En las primeras horas posteriores al trauma la expansión de hematomas es la principal amenaza; en los días siguientes, otros mecanismos que incluyen la acumulación de agua, expansión de contusiones, pérdida de autorregulación e isquemia llevan a elevaciones en la presión intracraneal. Las consecuencias directas de la elevación de la presión intracraneal en el tejido cerebral pueden ser diferenciadas en general como mecánicas y vasculares. Cuando una lesión ocupante de espacio se desarrolla, se origina un gradiente de presión desde esta área y causará daño en el tejido cerebral, desviación de la línea media, y desplazamiento del tejido cerebral en dirección medial o caudal (herniación). La herniación del tejido cerebral es una verdadera emergencia que requiere tratamiento inmediato para prevenir daño irreversible y a menudo fatal del tronco cerebral. ${ }^{13}$

Por otro lado, los efectos vasculares de una presión intracraneal elevada son causados por una presión de perfusión cerebral dañada. La presión de perfusión cerebral es la fuerza motriz detrás del flujo sanguíneo cerebral, pero los niveles que se requieren para mantener un flujo adecuado varían entre pacientes. Conforme la presión de perfusión cerebral desciende, el flujo sanguíneo cerebral puede volverse insuficiente para mantener una adecuada perfusión del tejido cerebral y oxigenación. ${ }^{17}$ 
La isquemia promueve la aparición de edema citotóxico, y resulta en presiones intracraneales aún más elevadas. Los efectos adversos de la presión intracraneal incrementada y la poca presión de perfusión cerebral en la mortalidad y en el pronóstico a largo plazo se han documentado en diversos estudios. ${ }^{18,19}$

\section{MONITORIZACIÓN DE LA PRESIÓN INTRACRANEAL}

La práctica de monitorizar la presión intracraneal fue iniciada por el trabajo de Guillaume y Janni en Francia y Lundberg en Suecia. ${ }^{20,21}$ En 1951 en una revista francesa Guillaume y Janni reportaron su experiencia con mediciones continuas de la presión intracraneal. En 1960 el neurocirujano sueco Nils Lundberg reportó una serie de casos de pacientes con tumores cerebrales en quienes monitorizó la presión intracraneal a través de derivaciones ventriculares externas. Más tarde, en 1965 se aplicó la experiencia de Lundberg en medir la presión intracraneal en pacientes con trauma craneoencefálico severo y se describió una serie de 30 casos de pacientes, los cuales fueron exitosamente monitorizados. ${ }^{22,23}$

Desde 1960 con Lundberg, la derivación ventricular externa se considera el estándar de oro para el monitoreo de la presión intracraneal. ${ }^{13}$

La elevación de la PIC es un fenómeno frecuente en los pacientes con trauma craneoencefálico, en el TCE grave se reporta entre 50 y $75 \%$ de los pacientes. ${ }^{10}$

La decisión de monitorizar la PIC se basa en evaluar las probabilidades de que el paciente en algún momento evolutivo pudiera presentar hemorragia intracerebral (HIC) determinada por la evolución del daño inicial, o por otras lesiones cerebrales o extracerebrales. La evaluación del riesgo de padecer HIC puede basarse en una combinación de características clínicas (coma, TCE grave) y de hallazgos en la tomografía computarizada (TC) inicial (desvío de la línea media, lesiones ocupantes de espacio $>25 \mathrm{~cm}^{3}$, compresión o ausencia de las cisternas perimesencefálicas, signos compatibles con hidrocefalia, entre otros). ${ }^{24}$ Las indicaciones para iniciar la monitorización de la presión intracraneal se resumen en la Tabla 1.
Los objetivos generales del neuromonitoreo son: 1) identificar el agravamiento de las funciones neurológicas y daño cerebral secundario que se beneficiaría de un tratamiento específico; 2) mejorar la comprensión de los mecanismos fisiopatológicos de las enfermedades neurológicas en los pacientes críticos; 3) proveer de datos fisiopatológicos claros que guíen las metas terapéuticas; 4) asistir en el pronóstico de los pacientes. ${ }^{24}$

Es importante recordar que no es el monitoreo per se lo que mejora el resultado de los pacientes, sino el uso de la información que se obtiene del monitoreo en el tratamiento. El tratamiento guiado por los resultados del monitoreo es mejor que un tratamiento guiado sólo por la evaluación clínica. ${ }^{25}$

Para el monitoreo de la presión intracraneal existen métodos invasivos y no invasivos. Entre los métodos invasivos están las derivaciones ventriculares externas y los catéteres para medición que se colocan directamente en el parénquima cerebral. De los métodos no invasivos existen la medición por ultrasonografía Doppler de los índices de pulsatilidad de las arterias cerebrales y la medición por ultrasonido de la vaina del nervio óptico.

\section{MEDICIÓN DEL DIÁMETRO DE LA VAINA DEL NERVIO ÓPTICO}

El nervio óptico es el segundo de los nervios craneales. Un tracto de fibras con aproximadamente $1.2 \mathrm{mi}-$ llones de axones, rodeado de vainas meníngeas que transportan las sensaciones visuales al sistema nervioso central. Está formado por los axones de las células ganglionares y por glía.

Se desprende del globo ocular $3 \mathrm{~mm}$ medialmente y 1 $\mathrm{mm}$ inferiormente al polo posterior del mismo. Se dirige posteromedialmente, atraviesa la cavidad craneal y termina en el ángulo anterolateral correspondiente al quiasma óptico. Su longitud total es de alrededor de $5 \mathrm{~cm}$.

Consta de cuatro tramos o segmentos:

- Primer segmento: intraocular/ intraescleral (1 mm).

- Segundo segmento: intraorbitario $(30 \mathrm{~mm})$.

Tabla 1: Indicaciones para monitorización de la presión intracraneal en pacientes con trauma craneoencefálico. ${ }^{24}$

\begin{tabular}{|c|c|c|}
\hline TCE grave & TCE moderado & TCE otros casos \\
\hline
\end{tabular}

TAC = tomografía axial computarizada; TCE = trauma craneoencefálico; GCS = escala de coma de Glasgow.

* Según la clasificación de las lesiones cerebrales por TAC. 
Consta de fibras mielinizadas rodeadas de vainas meníngeas (leptomeninges, duramadre y espacio subaracnoideo). Así, el nervio queda comprendido en un cono formado por los músculos rectos del ojo (cono muscular) y se sumerge en la grasa retroocular. En este segmento el nervio se relaciona con la arteria oftálmi$\mathrm{ca}$, las venas oftálmicas superior e inferior, los nervios nasociliar, oculomotor y abducens, el ganglio ciliar, los vasos y nervios ciliares. Su trayecto es ligeramente sinuoso en «S» itálica, lo que le permite adaptarse a los movimientos del ojo sin sufrir tensiones. Esta porción que forma una «S» es la que interesa para la evaluación del DVNO.

- Tercer segmento: intracanalicular.

El nervio atraviesa el foramen óptico acompañado de la arteria oftálmica (cara inferior).

- Cuarto segmento: intracraneal $(1 \mathrm{~cm})$.

Está comprendido entre el foramen óptico y el quiasma óptico. Se relaciona inferiormente con la tienda de la hipófisis y el canal óptico del esfenoides; superiormente con la arteria cerebral anterior y la estría olfatoria medial y lateralmente con la carótida interna. ${ }^{26}$

La vaina del nervio óptico (VNO) se encuentra en continuidad con la duramadre del cerebro. La vaina del nervio óptico envuelve el segmento intraorbitario del nervio óptico y se encuentra separado de él por una capa de líquido cerebroespinal (LCE) y trabécula aracnoidea. Este espacio subaracnoideo está conectado con la capa interna de la aracnoides, y los estudios en cadáver han demostrado una relación lineal entre la PIC y la presión perióptica del LCE. Una elevación de la PIC causa distensión de la vaina del nervio óptico, principalmente en la porción retrobulbar, dado que ésta posee una red trabecular menos densa. A medida que la PIC se eleva, el LCR se distribuye por la duramadre hacia la VNO, la cual ocasiona aumento en su diámetro. ${ }^{27}$

Algunas condiciones médicas pueden bloquear la circulación de LCE impidiendo la transmisión de la presión intracraneal a la vaina del nervio óptico como tumores cerebrales, edema cerebral con efecto de masa, tumores de la vaina del nervio óptico, anomalías vasculares, traumatismo ocular y orbital. ${ }^{28}$

La aplicación del ultrasonido a la práctica clínica cotidiana y a la cabecera del enfermo se ha venido posicionando como una excelente herramienta en diferentes escenarios de la medicina intensiva. Su implementación en el enfermo neurológico grave es parte integral del abordaje, destacando la medición ultrasonográfica del diámetro de la vaina del nervio óptico (DVNO), que en poco tiempo ha venido consolidándose como una nueva herramienta del neuromonitoreo. ${ }^{29,30}$

Tenon describió en 1806 la vaina de nervio óptico (VNO) como un recubrimiento conformado de LCR y meninges que envuelve al nervio óptico y a la esclera del globo ocular, pero fue hasta 1867 cuando Luschka demostró que la capa fibrosa que recubre al nervio óptico es una comunicación de la porción interna de la duramadre. ${ }^{31}$

En 1997 Hansen y Helmke reportaron por primera vez el uso de la ultrasonografía en modo B para medir el diámetro de la vaina del nervio óptico con el fin de evaluar a los pacientes con hipertensión intracraneal. Compararon el diámetro de la vaina del nervio óptico medido en 16 pacientes con traumatismo craneoencefálico severo agudo contra controles sanos y encontraron diferencia significativa en la media del diámetro de la vaina del nervio óptico. Descubrieron que un diámetro de la vaina del nervio óptico mayor de $5 \mathrm{~mm}$ tenía sensibilidad de $100 \%$ para detectar hipertensión intracraneal en su cohorte. Observaron además que el diámetro de la vaina del nervio óptico aumenta su tamaño rápidamente cuatro horas posteriores al trauma. Concluyeron que la medición de la vaina del nervio óptico tiene un alto grado de precisión para detectar hipertensión intracraneal. ${ }^{30,32}$

Se ha demostrado que la medición del DVNO con ultrasonografía tiene una curva de aprendizaje pequeña, y un adiestramiento sencillo del uso del equipo, lo que puede ser suficiente para un monitoreo dinámico. Ballantyne y asociados corroboran lo anterior en su estudio, en el cual un experto radiólogo en ultrasonografía midió la vaina en 76 pacientes sanos, luego la compararon con personas que iniciaban el adiestramiento, demostrando una variación en la medición de 0.2 milímetros, lo que confirma que esta técnica de monitoreo es fácilmente reproducible y que puede aplicarse en una UTI o en un servicio de urgencias. ${ }^{29,33}$

La medición se realiza con un transductor lineal (onda de 5-10 MHz) que se coloca sobre el párpado del paciente. Se recomienda no hacer presión directa sobre el globo ocular para evitar que se presente desprendimiento de retina o incremento de la presión intraocular. Con esta recomendación, se hace la búsqueda del nervio óptico (NO); una vez encontrado, se realiza la primera medición en la unión de la retina y el NO, contándose $0.3 \mathrm{~cm}$ de forma transversal; subsecuentemente se traza una línea perpendicular y se hace la medición de la vaina (Figura 1). Tiene una sensibilidad y especificidad para la detección de hipertensión intracraneal de 88 y $93 \%$, respectivamente, tomando como punto de corte mayor o igual a $0.5 \mathrm{~cm}(5 \mathrm{~mm})$, lo que se traduce como una PIC > $20 \mathrm{mmHg} .{ }^{34-38}$

El aumento del diámetro de la vaina del nervio óptico por tomografía simple de cráneo se ha validado en diversos estudios. En un estudio de 2014 se demostró una asociación lineal entre la medición por tomografía de la vaina del nervio óptico y la presión intracraneal medida por un método invasivo en pacientes con trauma craneoencefálico severo. El diámetro de la VNO mostró una asociación excelente para el mismo 


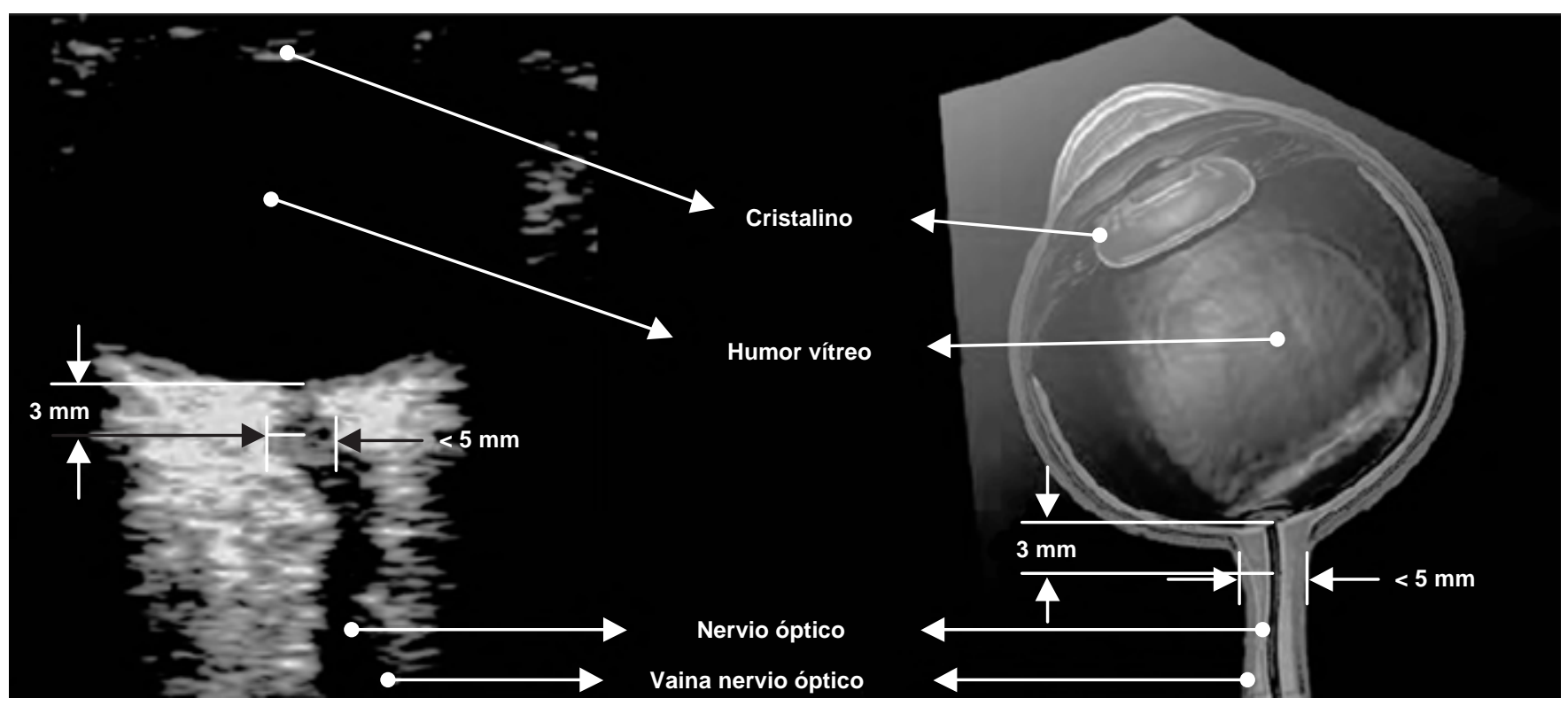

Figura 1: Imagen en la que se observa la técnica de medición del diámetro de la vaina del nervio óptico.

paciente, además de habilidad para discriminar entre los pacientes con hipertensión intracraneal. Por último, en este estudio se demostró que la medición del diámetro de la vaina del nervio óptico es un predictor del aumento de la presión intracraneal mucho más fuerte que las características que comúnmente se buscan en las tomografías de cráneo (borramiento de las cisternas, herniación cerebral, ocupación ventricular, etcétera). ${ }^{39}$

Recientemente se han calculado las variaciones interobservador e intraobservador de las mediciones por ultrasonido del diámetro de la VNO en 0.5 y $0.2 \mathrm{~mm}$ respectivamente, estos resultados son muy similares a los reportados para las mediciones por tomografía simple de cráneo de la vaina del nervio óptico. ${ }^{40}$

\section{MATERIAL Y MÉTODOS}

El objetivo de nuestro estudio fue comparar las mediciones del diámetro de la vaina del nervio óptico a 3, 6 y 9 $\mathrm{mm}$ mediante ultrasonografía contra las medidas obtenidas a las mismas distancias por tomografía simple de cráneo y así encontrar en cuál de las tres distancias se presenta menor diferencia entre ambas mediciones en pacientes con trauma craneoencefálico grave.

Debido a la trayectoria relativamente larga del nervio óptico se pretende determinar el mejor sitio para realizar la medición de la vaina del nervio óptico, dado que éste presenta durante su trayecto varios acodamientos o curvas que pueden sesgar la medición correcta del diámetro de la vaina del nervio óptico.

Se realizó un estudio de tipo observacional, prospectivo, transversal y comparativo en pacientes con diagnóstico de trauma craneoencefálico grave que in- gresaron a la unidad de cuidados intensivos de nuestro hospital. Los pacientes fueron seleccionados con base en los siguientes criterios. Todos los pacientes que ingresaron al servicio de terapia intensiva neurológica con diagnóstico de trauma craneoencefálico grave definido por el puntaje de la escala de coma de Glasgow (menor de ocho puntos) asignado por el servicio de urgencias a su ingreso, con tiempo transcurrido entre la medición por tomografía simple de cráneo y por ultrasonido menor de una hora. Todos los pacientes debían contar con ambos globos oculares íntegros, sin antecedentes conocidos de glaucoma, con integridad de huesos del cráneo y pacientes cuyos familiares firmaron el formato de consentimiento informado de ingreso a terapia intensiva. Fueron descartados aquellos pacientes en los que no se encontró una adecuada ventana para realizar el estudio por ultrasonografía de la vaina del nervio óptico, los pacientes cuyo reporte de medición de la vaina del nervio óptico por tomografía simple de cráneo no se recibió por parte del servicio de radiología.

La medición por ultrasonografía del diámetro de la vaina del nervio óptico se llevó a cabo en modo bidimensional a $3 \mathrm{~mm}$ detrás de la retina, una vez localizado este punto se trazó una línea horizontal de borde a borde de la vaina del nervio óptico, es decir, del borde externo hiperecogénico de la duramadre al borde externo hiperecogénico del otro lado de la duramadre, la cual se midió en milímetros, encontrando en medio una estructura hipoecoica de trayecto regular posterior al globo ocular, realizándose el mismo procedimiento a las distancias de 6 y $9 \mathrm{~mm}$. El paciente se colocó en posición supina con elevación de la cabeza a $20-30^{\circ}$ con el transductor en posición horizontal. Se consideró 
adecuada una vaina del nervio óptico si se lograba una visualización a $9 \mathrm{~mm}$ por detrás del globo ocular y la órbita no estaba oscurecida por estructuras cercanas. Cabe señalar que de forma tradicional la medición del diámetro de la vaina del nervio óptico es un promedio de la medición en plano horizontal y en plano vertical; sin embargo, ya que por tomografía simple de cráneo sólo se obtiene la medición en el plano horizontal, de igual forma sólo se realizó la medición por ultrasonido en el plano horizontal.

Por parte del servicio de radiología se obtuvieron mediciones por tomografía simple de cráneo de los nervios ópticos a 3, 6 y $9 \mathrm{~mm}$ de la unión con la retina en cortes transversales de todos los pacientes.

Con base en las variables del estudio y previa codificación, se diseñó la base de datos en el paquete SPSS ${ }^{\circledR}$ y en el Software Excel, ${ }^{\circledR}$ para obtener las frecuencias se elaboraron cuadros de presentación así como gráficos en función del tipo de escala y variable. Se calcularon las medidas de tendencia central y las de dispersión para las variables cuantitativas, y para las cualitativas se utilizaron porcentajes y cifras absolutas, comparando los resultados de las mediciones obtenidas a través de ultrasonido (mediciones de nervio óptico) y de tomografía en ojo derecho e izquierdo para de- terminar la existencia o no existencia de «significancia estadística de las diferencias», aplicando la prueba «T» de Student, adoptando $p<0.05$.

\section{RESULTADOS}

La mayor cifra de pacientes estudiados se presentó en el grupo de 30 a 34 años de edad, significando $20.83 \%$ sobre el total de los 24 analizados; por otra parte, el grupo de 20 a 24 años de edad representó $12.50 \%$; y en tercer lugar el grupo de 45 a 49 años de edad con $8.33 \%$; sin embargo, $62.50 \%$ de los pacientes con trauma craneoencefálico fueron menores de 50 años de edad. Se observó en el sexo masculino un promedio o media menor con respecto al promedio de las mujeres; 35.39 vs. 39.83; lo anterior se refleja en mayor dispersión también en las mujeres, lo cual se observa en la cifra de desviación estándar, el resultado de la diferencia es «no significativo» con $p>0.05$; sin embargo, con respecto al coeficiente de variación (desviación estándar/media o promedio) la cifra es mayor en los hombres. El intervalo estimado para un nivel de $95 \%$ de confianza fue el siguiente: hombres 17-64.51 y mujeres 19-71.81. De los pacientes estudiados $75 \%$ correspondieron al sexo masculino, y $25 \%$ al femenino.

Tabla 2: Indicadores del resumen de la medida del diámetro de la vaina del nervio óptico por ultrasonografía y por tomografía computarizada a 3, 6 y $9 \mathrm{~mm}$ de la retina en pacientes con traumatismo craneoencefálico.

\begin{tabular}{|c|c|c|c|c|}
\hline Indicadores & \multicolumn{2}{|c|}{ Ojo derecho } & \multicolumn{2}{|c|}{ Ojo izquierdo } \\
\hline \multicolumn{5}{|l|}{ Medición a 3 mm } \\
\hline Media & 5.15 & 5.19 & 5.38 & 5.57 \\
\hline Mediana & 5.25 & 5.30 & 5.15 & 5.75 \\
\hline Moda & 5.40 & 6.20 & 4.90 & 6.20 \\
\hline $\mathrm{DE}$ & 0.79 & 0.88 & 0.87 & 1.21 \\
\hline CV & 15.34 & 16.96 & 16.17 & 21.72 \\
\hline IC & $3.57-6.73$ & $3.43-6.95$ & $3.64-7.12$ & $3.15-7.99$ \\
\hline \multicolumn{5}{|l|}{ Medición a 6 mm } \\
\hline Media & 5.30 & 5.01 & 5.76 & 5.10 \\
\hline Mediana & 5.30 & 5.10 & 5.75 & 5.05 \\
\hline Moda & 6.10 & 6.20 & 6.70 & 4.20 \\
\hline IC & $3.54-7.06$ & $3.11-6.91$ & $3.88-7.64$ & $3.00-7.20$ \\
\hline \multicolumn{5}{|l|}{ Medición a 9 mm } \\
\hline Media & 4.67 & 4.60 & 5.65 & 4.85 \\
\hline Mediana & 5.35 & 4.60 & 5.80 & 4.60 \\
\hline Moda & 6.70 & 5.80 & 6.20 & 5 \\
\hline $\mathrm{DE}$ & 0.76 & 0.87 & 0.98 & 1.11 \\
\hline Cifra mínima & 4.10 & 3 & 3.60 & 3.10 \\
\hline Cifra máxima & 6.70 & 6.20 & 7.10 & 7 \\
\hline CV & 16.27 & 18.91 & 17.35 & 22.89 \\
\hline IC & $3.15-6.19$ & $2.86-6.34$ & $3.69-7.61$ & $2.63-7.07$ \\
\hline
\end{tabular}

$\mathrm{DE}$ = desviación estándar; $\mathrm{CV}$ = coeficiente de variación; $\mathrm{IC}$ = intervalo de confianza. 
Tabla 3: Promedios del nervio óptico con ultrasonido y tomografía en pacientes con trauma craneoencefálico.

\begin{tabular}{lccc}
\hline Indicadores & Ultrasonido & $\begin{array}{c}\text { Tomografía axial } \\
\text { computarizada }\end{array}$ & Diferencias \\
\hline Ojo derecho $(\mathrm{mm})$ & & & \\
3 & 5.15 & 5.19 & 0.04 \\
6 & 5.30 & 5.01 & 0.30 \\
9 & 4.67 & 4.60 & 0.07 \\
Ojo izquierdo $(\mathrm{mm})$ & & & \\
3 & 5.38 & 5.57 & 0.19 \\
6 & 5.76 & 5.10 & 0.66 \\
9 & 5.65 & 4.85 & 0.80 \\
\hline
\end{tabular}

Del lado derecho a $3 \mathrm{~mm}$ se observó un promedio o media menor con ultrasonografía respecto al promedio de tomografía computarizada; 5.15 vs. 5.19 ; lo anterior se refleja en mayor dispersión también en el resultado obtenido con la tomografía, lo cual se observa en la cifra de desviación estándar, el resultado de la diferencia es «no significativo» con $p>0.05$; sin embargo, con respecto al coeficiente de variación (desviación estándar/media o promedio) la cifra también es mayor en el resultado obtenido con tomografía computarizada. EI intervalo estimado para un nivel de $95 \%$ de confianza fue el siguiente: ultrasonografía 3.57-6.73 y tomografía computarizada 3.43-6.95 (Tablas 2 y 3).

Del mismo lado, pero a $6 \mathrm{~mm}$, se observó un promedio o media mayor con ultrasonografía respecto al promedio de tomografía computarizada; 5.30 vs. 5.01 ; se refleja mayor dispersión en el resultado con tomografía computarizada, lo cual se observa en la cifra de desviación estándar, el resultado de la diferencia es «no significativa» con $p>0.05$; sin embargo, con respecto al coeficiente de variación (desviación estándar/media o promedio) la cifra también es mayor en el resultado obtenido con tomografía computarizada. El intervalo estimado para un nivel de $95 \%$ de confianza fue el siguiente: ultrasonografía 3.54-7.06 y tomografía computarizada 3.11-6.91 (Tablas 2 y 3).

Por último, a $9 \mathrm{~mm}$ se observó un promedio o media mayor con ultrasonografía respecto al promedio de tomografía computarizada; 4.67 vs. 4.60 ; se refleja mayor dispersión en el resultado con tomografía computarizada, lo cual se observa en la cifra de desviación estándar, el resultado de la diferencia es "no significativa" con $p>0.05$; sin embargo, con respecto al coeficiente de variación (desviación estándar/media o promedio) la cifra también es mayor en el resultado obtenido con tomografía computarizada. El intervalo estimado para un nivel de $95 \%$ de confianza fue el siguiente: ultrasonografía 3.15-6.19 y tomografía computarizada 2.86-6.34 (Tablas 2 y 3 ).

De la misma forma, del lado izquierdo a $3 \mathrm{~mm}$ se observó un promedio o media mayor con tomografía computarizada respecto al promedio de ultrasonografía;
5.57 vs. 5.38; se refleja mayor dispersión en el resultado con tomografía computarizada, lo cual se observa en la cifra de desviación estándar, el resultado de la diferencia es «no significativa» con $p>0.05$; sin embargo, con respecto al coeficiente de variación (desviación estándar/media o promedio) la cifra también es mayor en el resultado obtenido con tomografía computarizada. El intervalo estimado para un nivel de $95 \%$ de confianza fue el siguiente: ultrasonografía 3.64-7.12 y tomografía computarizada 3.15-7.99 (Tablas 2 y 3).

A $6 \mathrm{~mm}$ del lado izquierdo se observó un promedio o media mayor con ultrasonografía respecto al promedio de tomografía computarizada; 5.76 vs. 5.10; se refleja mayor dispersión en el resultado con tomografía computarizada, lo cual se observa en la cifra de desviación estándar, el resultado de la diferencia es «no significativa» con $p>0.05$; sin embargo, con respecto al coeficiente de variación (desviación estándar/media o promedio) la cifra también es mayor en el resultado obtenido con tomografía computarizada. El intervalo estimado para un nivel de $95 \%$ de confianza fue el siguiente: ultrasonografía 3.88-7.64 y tomografía computarizada 3.00-7.20 (Tablas 2 y 3).

Por último, a $9 \mathrm{~mm}$ se observó un promedio o media mayor con ultrasonografía respecto al promedio de tomografía computarizada; 5.65 vs. 4.85 ; se refleja mayor dispersión en el resultado con tomografía computarizada, lo cual se observa en la cifra de desviación estándar, el resultado de la diferencia es «no significativa» con $p$ $>0.05$; sin embargo, con respecto al coeficiente de variación (desviación estándar/media o promedio) la cifra también es mayor en el resultado obtenido con tomografía computarizada. El intervalo estimado para un nivel de 95\% de confianza fue el siguiente: ultrasonografía 3.697.61 y tomografía computarizada 2.63-7.07 (Tablas 2 y 3).

El principal hallazgo obtenido a partir de la tomografía fue la hemorragia subaracnoidea, representando $50 \%$ en relación al total de 24 pacientes que integraron la muestra estudiada y en segundo sitio, el hematoma subdural con $37.50 \%$.

\section{DISCUSIÓN}

La medición por ultrasonido del diámetro de la vaina del nervio óptico es un método de neuromonitoreo con una curva de aprendizaje relativamente pequeña para los profesionales de medicina del enfermo en estado crítico. Tiene una buena relación costo-beneficio en comparación con el monitoreo que utiliza la tomografía. El ultrasonido tiene además la ventaja de poderse llevar a cabo a la cabecera del paciente con pocas contraindicaciones y efectos adversos.

La validación de este método de neuromonitoreo se ha llevado a cabo mediante grandes metaanálisis. Sin embargo, al revisar la literatura sobre el tema no se en- 
contraron antecedentes de estudios que comparen la medición por ultrasonido de la vaina del nervio óptico a diferentes distancias vs. validación con tomografía simple de cráneo.

Además, la medición de la vaina del nervio óptico como método de neuromonitoreo es un recurso fácilmente asequible, lo que lo convierte en una excelente herramienta para el control de los pacientes críticos neurológicos que tengan riesgo de desarrollar hipertensión intracraneal. Los estudios que existen sobre la técnica reportan que el sitio para la medición debe realizarse a $3 \mathrm{~mm}$ por detrás del globo ocular. Sin embargo, es en este mismo punto anatómico donde el nervio óptico tiene un acodamiento y podría llegar a realizarse la medición en forma oblicua a su eje vertical, por lo que este error podría ser una causa de sesgo en la medición.

El nervio óptico en su porción infraorbitaria mide aproximadamente $30 \mathrm{~mm}$, y el aumento de la presión intracraneal se transmite de forma uniforme a toda su extensión.

La aplicación del ultrasonido a la práctica clínica cotidiana y a la cabecera del enfermo se ha posicionado como una excelente herramienta en diferentes escenarios de la medicina intensiva. Su implementación en el manejo del enfermo neurológico grave puede considerarse como parte integral del abordaje, destacando la medición ultrasonográfica del diámetro de la vaina del nervio óptico, que en poco tiempo se ha consolidado como una sólida herramienta del neuromonitoreo. ${ }^{28,29}$

A pesar de que los resultados de la investigación realizada son fundamentales para el manejo clínico de los pacientes con traumatismo craneoencefálico, se considera necesario que se desarrollen acciones de prevención con el objetivo de disminuir su frecuencia, debido a que se le considera un problema de salud pública, sobre todo en población económicamente activa con las repercusiones que ello implica. Las acciones preventivas deben enfocarse en la detección de los factores de riesgo, esto unido al manejo clínico adecuado y a la accesibilidad a la rehabilitación puede mejorar el pronóstico del traumatismo craneoencefálico.

\section{CONCLUSIONES}

De acuerdo con lo planeado para llevar a la práctica la investigación y obtención de los datos se determinaron, por parte del servicio de radiología, mediciones a través de tomografía simple de cráneo de los nervios ópticos a 3, 6 y $9 \mathrm{~mm}$ de la unión con la retina en cortes transversales de los 24 pacientes que integraron la muestra. Para su medición las imágenes de las tomografías simples de cráneo fueron desplegadas en un visualizador digital. Las imágenes se amplificaron donde aparece el nervio óptico en su eje más largo y con el diámetro más amplio. Se utilizó la ventana para abdomen (unidades
Hounsfield en un rango de 25 a 40), la cual demostró la vaina del nervio óptico en mejor detalle.

Concretamente, se obtuvieron las cifras a partir de las mediciones del diámetro de la vaina del nervio óptico a 3, 6 y $9 \mathrm{~mm}$ mediante ultrasonido, se compararon con los resultados a las mismas distancias mediante la tomografía simple de cráneo en pacientes con traumatismo craneoencefálico grave atendidos en la unidad de cuidados neurointensivos. En ningún caso se registró «diferencia estadísticamente significativa» a favor de alguna de las técnicas e instrumentos utilizados.

Se obtuvo información de la edad, género, diagnóstico por tomografía simple de cráneo y Glasgow de ingreso, estos datos se presentan en tablas y gráficos que ilustran objetivamente las cifras absolutas y relativas. Con respecto a Glasgow, los 24 pacientes que tuvieron cifras $\leq 8$ se clasificaron como «graves». Por otra parte, el principal hallazgo obtenido por tomografía fue la hemorragia subaracnoidea presentada en la mitad de los pacientes analizados.

\section{AGRADECIMIENTOS}

Nuestro agradecimiento por su colaboración a los doctores:

Víctor Hugo Salas Bautista, Técnico Radiólogo adscrito al Servicio de Radiología e Imagen, Hospital General Ecatepec Las Américas del Instituto de Salud del Estado de México.

Sócrates Orozco Ramírez, Médico Radiólogo, Jefe del Servicio de Radiología e Imagen, Hospital General Ecatepec Las Américas del Instituto de Salud del Estado de México.

\section{BIBLIOGRAFÍA}

1. Stocchetti N, Carbonara M, Citerio G, Ercole A, Skrifvars M, et al. Severe traumatic brain injury: targeted management in the intensive care unit. Lancet Neurol. 2017;16:452-464.

2. Jallo J, Loftus C. Neurotrauma and critical care of the brain. 2nd ed. New York: Thieme Medical Publishers; 2018.

3. Carrillo-Esper R, Meza-Márquez JM. Trauma craneoencefálico. Rev Mex Anest. 2015;38(Suppl: 3):433-434.

4. Hamdan G. Trauma craneoencefálico severo: Parte I. Medicrit. 2005;2(7):107-148.

5. Advanced trauma life support. 10th ed. Chicago, IL: American College of Surgeons; 2018.

6. Bulger EM, Nathens AB, Rivara FP, et al. Management of severe head injury: institutional variations in care and effect on outcome. Crit Care Med. 2002;30:1870-1876.

7. Estrada F, Morales J, Tabla E, Solís B, Navarro H, et al. Neuro protección y traumatismo craneoencefálico. Rev Fac Med UNAM. 2012;55(4):16-29.

8. Alali AS, Temkin N, Barber J, et al. A clinical decision rule to predict intracranial hypertension in severe traumatic brain injury. $J$ Neurosurg. 2018;131(2):612-619.

9. Part 2: Prognosis in penetrating brain injury. J Trauma. 2001;51(2 Suppl):S44-S86.

10. Mori LB, Díaz MF, Dorfam B, Sociedad Argentina de Terapia Intensiva; et al. Neurointensivismo: enfoque clínico, diagnóstico y terapéutica. Buenos Aires: Médica Panamericana; 2010. 
11. Aguirre Rodríguez JI. Capítulo 61: Flujo sanguíneo cerebral, líquido cefalorraquídeo y metabolismo cerebral. En: Guyton AC, Hall JE. Tratado de fisiología médica. 10a ed. España: McGrawHill; 2000. pp. 855-862.

12. Schoser BG, Riemenschneider N, Hansen HC. The impact of raised intracranial pressure on cerebral venous hemodynamics: a prospective venous transcranial Doppler ultrasonography study. J Neurosurg. 1999;91(5):744-749.

13. Finfer $\mathrm{S}$, Vincent J. Traumatic intracranial hypertension. N Engl J Med. 2014;370:2121-2130.

14. Balestreri M, Czosnyka M, Hutchinson P, Steiner LA, Hiler M, Smielewski $P$, et al. Impact of intracranial pressure and cerebral perfusion pressure on severe disability and mortality after head injury. Neurocrit Care. 2006;4:8-13. https://doi.org/10.1385/NCC:4:1:008

15. Badri S, Chen J, Barber J, Temkin NR, Dikmen SS, Chesnut RM, et al. Mortality and long-term functional outcome associated with intracranial pressure after traumatic brain injury. Intensive Care Med. 2012;38:1800-1809. https://doi.org/10.1007/s00134-0122655-4

16. Stocchetti $N$, Mass $A$. Traumatic intracranial hypertension. $N$ Engl J Med. 2014;370:2121-2130.

17. Nordström $\mathrm{CH}$, Reinstrup P, Xu W, Gardenfors A, Ungerstedt U. Assessment of the lower limit for cerebral perfusion pressure in severe head injuries by bedside monitoring of regional energy metabolism. Anesthesiology. 2003;98:809-814.

18. Stocchetti N, Zanaboni C, Colombo A, et al. Refractory intracranial hypertensionand "second-tier" therapies in traumatic brain injury. Intensive Care Med. 2008;34:461-467.

19. Vik A, Nag T, Fredriksli OA, et al. Relationship of "dose" of intracranial hypertension to outcome in severe traumatic brain injury. J Neurosurg. 2008;109:678-684.

20. Guillaume J, Janny P. Manométrie intracrânienne continue: intérêt de la méthode et premiers résultats. Rev Neurol (Paris). 1951;84:131-142.

21. Lundberg N. Continuous recording and control of ventricular fluid pressure in neurosurgical practice. Acta Psychiatr Scand Suppl. 1960;36(149):1-193.

22. Ryder HW, Espey FF, Kimbell FD, et al. The mechanism of the change in cerebrospinal fluid pressure following an induced change in the volume of the fluid space. J Lab Clin Med. 1953;41(3):428-435.

23. Lundberg $\mathrm{N}$, Troupp $\mathrm{H}$, Lorin $\mathrm{H}$. Continuous recording of the ventricular-fluid pressure in patients with severe acute traumatic brain injury. A preliminary report. J Neurosurg. 1965;22(6):581-590.

24. Stocchetti N, Le Roux P, Vespa P, Oddo M, Citerio G, et al. Clinical review: neuromonitoring- an update. Crit Care. 2013;17(1):201.

25. Carney N, Totten AM, O'Reilly C, et al. Guidelines for the management of severe traumatic brain injury, fourth edition. Neurosurgery. 2017;80(1):6-15.

26. Muñoz Hernández AM, Santos Bueso E. Nervio óptico. Anatomía y fisiología. Boletín Soc Oftalmo Madrid. 2013;53.

27. Ohle R, Mclsaac S, Woo M, Perry J. Sonography of the optic nerve sheath diameter for detection of raised intracranial pressure compared to computed tomography. J Ultrasound Med. 2015;34(7):1285-1294.

28. Rincón J. Manual de ultrasonido en terapia intensiva y emergencias. 2a edición. Madrid: Editorial Prado; 2016.

29. Carrillo R, Rojo O, Cruz J, Romero J. Diámetro de la vaina del nervio óptico. Una herramienta para el monitoreo dinámico de la hipertensión intracraneana. Rev Asoc Mex Med Crit Ter Int. 2016;30(4):249-252.
30. Geeraerts T, Merceron S, Benhamou D, Vigué B, Duranteau J. Noninvasive assessment of intracranial pressure using ocular sonography in neurocritical care patients. Intensive Care Med. 2008;34:2062-2067.

31. Dubost C, Motuel J, Geeraerts T. Mesure de la pression intracranienne sans capteur: comment et pour qui, non-invasive evaluation of intracranial pressure: how and for whom. Ann Fr Anesth Reanim. 2012;31(6):125-132.

32. Geeraerts T, Launey Y, Martin L, et al. Ultrasonography of the optic nerve sheath may be useful for detecting raised intracranial pressure after severe brain injury. Intensive Care Med. 2007;33(10):1704-1711.

33. Ballantyne SA, Neill GO, Hamilton R, Hollman AS. Observer variation in the sonographic measurement of optic nerve sheath diameter in normal adults. Eur J Ultrasound. 2002;15:145-149.

34. Zepeda A, Carrillo R. Medición ultrasonográfica del diámetro de la vaina del nervio óptico como marcador de hipertensión intracraneana. Rev Mex Anestesiol. 2017;40(1): 255-257.

35. Ochoa L, Cardozo A. Aplicaciones de la ultrasonografía en el sistema nervioso central para neuroanestesia y cuidado neurocrítico. Rev Colomb Anestesiol. 2015;43(4):314-320.

36. Blaivas M, Theodoro D, Sierzenski PR. Elevated intracranial pressure detected by bedside emergency ultrasonography of the optic nerve sheath. Acad Emerg Med. 2003;10(4):376-381.

37. Rajajee V, Fletcher JJ, Rochlen LR, Jacobs TL. Comparison of accuracy of optic nerve ultrasound for the detection of intracranial hypertension in the setting of acutely fluctuating vs stable intracranial pressure: post-hoc analysis of data from a prospective, blinded single center study. Crit Care. 2012;16(3):R79.

38. Kalantari H, Jaiswal R, Bruck I, Matari H, Ghobadi F, et al. Correlation of optic nerve sheath diameter measurements by computed tomography and magnetic resonance imaging. $A m \mathrm{~J}$ Emerg Med. 2013;31(11):1595-1597.

39. Sekhon M, Griesdale D, Robba C, McGlashan N, Needham E. Optic nerve sheath diameter on computed tomography is correlated with simultaneously measured intracranial pressure in patients with severe traumatic brain injury. Intensive Care Med. 2014;40:1267-1274.

40. Legrand A, Jeanjean P, Delanghe F, Johann P, Lecat B, Dupont $H$. Estimation of optic nerve sheath diameter on an initial brain computed tomography scan can contribute prognostic information in traumatic brain injury patients. Crit Care. 2013;17(2): R61. doi: 10.1186/cc12589.

Conflicto de intereses: Los autores declaran no tener conflicto de intereses en relación con el presente artículo. Financiamiento: Los autores declaran la no existencia de financiamiento en relación al presente artículo.

\section{Correspondencia:}

Roberto Emmanuel Islas Ávila

Simón Bolívar S/N, esquina

Libertadores de América,

Lote I, Manzana 10, Fraccionamiento Las Américas,

55076, Ecatepec de Morelos, Estado de México.

E-mail: hakym9@gmail.com 